\title{
Diagnóstico en Educación de los Trastornos de Espectro Autista
}

\section{Autism Spectrum Disorders Diagnosis in Educational Contexts}

Fecha de recepción: 08/01/2016

Fecha de aceptación: 11/01/2016

\author{
Aranzazu Cervera Chulvi, Sonia Martínez-Sanchis, \\ Ricard Marí Molla y Jesús Suárez Rodríguez \\ Universitat de València
}

\section{resumen/abstract:}

Este artículo expone las conclusiones de consenso de diferentes guías de buenas prácticas nacionales e internacionales como referente para la práctica profesional del diagnóstico de los TEA en educación. El proceso incorpora 5 fases:Recogida de información, análisis de la información, valoración de la información, propuesta de intervención desde la que se valida el proceso diagnóstico y evaluación del conjunto del proceso, incluyendo a todos los agentes implicados. Barreras en la organización, así como políticas internas o externas, pueden dificultar la aplicación de las recomendaciones de las buenas prácticas. Resulta necesario profundizar en estos aspectos, así como en las causas e implicaciones de la separación entre diagnóstico e intervención para diseñar acciones destinadas a su integración.

This article exposes the main conclusions of different guides of national and international best practices as a referent for the professional practice of the diagnostic of ASD in education. The process of diagnostic would incorporate 5 phases: Collection of information, analysis of the information, assessment of the information, proposal of intervention which validates the process diagnostic and evaluation of the whole process, including the agents involved in it. Barriers in the organization as well as internal or external policies could difficult follow the recommendations of the best practices. It would be necessary to analyze this as well as the causes and implications of the separation between diagnostic and intervention to design actions leading to their integration.

\section{palabras clave/keywords:}

Trastornos de Espectro Autista, Diagnóstico, Educación, Buenas Prácticas

Autism Spectrum Disorders, Diagnosis, Education, Best practices,

\section{Introducción}

El debate sobre el diagnóstico de los Trastornos de Espectro Autista (TEA) parece estar irremediablemente unido a su epidemiología. Una prevalencia de 1 de cada 100 personas con TEA (Fernell, Eriksson, y Gillberg, 2013; Nygren et al., 2011; Simonoff, 2012), 1 de cada 68 según los últimos datos del Centers forDisease Control and Prevention (CDC, 2014), es argumento suficiente para emprender un abordaje en profundidad sobre los TEA, su evaluación e intervención. Pero desde la perspectiva de un profesional, basta con un solo caso para que este análisis sea una necesidad.

Siempre resulta difícil responder a una familia que pregunta “¿qué le pasa a mi hijo?”, pero ante los TEA la complejidad se multiplica. No sólo se trata de un espectro autista sino de diferentes espectros autistas (Fernell, Eriksson, y Gillberg, 2013). Rara vez se presentan aislados, coexisten con otros problemas neurológicos y de desarrollo; y la comorbilidad es 
la norma, no la excepción. Antes de los 5 años, son múltiples los síntomas compartidos por posteriores diagnósticos (Gillberg, 2010). Y, a falta de más avances, aún siendo un trastorno del neurodesarrollo (Balasubramanian, Bhatt, y Goyel, 2009; Blatt, 2010; Casey et al., 2012; Leblond et al., 2012; Zimmerman, 2008) los marcadores diagnósticos son conductuales, no biológicos.

En este escenario el profesional busca referentes válidos que le permitan mejorar su práctica. Pero la praxis, a su vez, se convierte también en objeto de investigación, puesto que un diagnóstico válido y fiable aumentará las posibilidades de intervención y el bienestar de las familias (Noterdaeme y Hutzelmeyer-Nickels, 2010; Volkmar y Pauls, 2003) y de las personas con TEA (Adelman, 2010; Canal-Bedia et al., 2015; Fountain, King, y Bearman, 2011; Klin, Klaiman y Jones, 2015).

Desde el enfoque de la calidad de la práctica diagnóstica, se han desarrollado diferentes guías clínicas o de buenas prácticas, basadas en el consenso de expertos (Diez-Cuervo et al., 2005) y en la evidencia científica (Filipeck et al., 2000; NAPC, 2003; NICE 2011; SIGN, 2007; SNS, 2009). Con el objetivo de guiar a los profesionales implicados en el diagnóstico de los TEA en edades pre-escolares y escolares, estos documentos recogen un conjunto de recomendaciones a modo de estándares de calidad desde un enfoque comprehensivo y multidisciplinar.

En términos generales, las propuestas de estas guías sitúan al diagnóstico a medio camino entre la categorización clasificatoria de un modelo médico y la de un modelo educativo dirigido a la definición de perfiles de desarrollo y necesidades educativas. Pero desde la educación, "el TEA es sólo uno de los aspectos a la hora de establecer las necesidades [...] el/ la niño/a debe ser considerado como un individuo no como una categoría. Las necesidades no son estáticas; disminuyen en aquellas situaciones en las que son bien atendidas y varían con las fortalezas del/la niño/a" (LeCouteur et al., 2003). De otro modo, el profesional encargado de realizar el diagnóstico en el campo educativo, se enfrenta a una realidad aún más compleja si cabe.

En este artículo expondremos las conclusiones de consenso de diferentes guías de buenas prácticas nacionales e internacionales, como referente para la práctica profesional del diagnóstico de los TEA en educación. Pero, este análisis es sólo un primer paso hacia una propuesta adaptada al ámbito educativo.

\section{Estándares de calidad desde las guías de buenas prácticas}

Como ya anunciábamos, los estándares establecidos para las buenas prácticas clínicas no puede ser trasladados, en principio, de forma exacta y directa para la práctica profesional en educación, sino que requiere de grandes dosis de cautela y adaptación. Pero, a falta de una guía basada en la evidencia desde el ámbito educativo (McKenney et al., 2015), constituyen un modelo de calidad válido para el proceso diagnóstico también desde la educación.

En este estudio seleccionaremos siete guías prácticas de referencia para el establecimiento de los principios de calidad de la praxis. Esta elección se basa en tres criterios básicos:

- Que aparezcan incluidas en los compiladores de guías clínicas basadas en la evidencia (NationalGuidelineClearinghouse, Guidelines International Network -GIN-, Nationale- 
lectronic Library for Medicines -NeLM-, GuiaSalud). Y que tomen como referencia los criterios del instrumento AGREE,que es una herramienta de evaluación de calidad de Guías de Práctica Clínica (véase http://www.agreetrust.org/home).

- Su autoría es atribuible al consenso de expertos y cuentan con el respaldo de grupos de investigación (como el Grupo de Estudio de los TEA del Instituto de Salud Carlos III), instituciones profesionales (American Academy of Neurology, American Academy of Pediatrics) o gubernamentales (Instituto Nacional de Seguridad Socialde New Zealand)

- Entre sus autores se recogen disciplinas y/o servicios con enfoque educativo como partícipes en la elaboración del documento o como profesionales reconocidos para el uso del mismo (Psicólogos, Pedagogos, Psicopedagogos, Maestros, Logopedas, etc.; Ministerios, Departamentos o Consejerías de Educación, Equipos especializados de Atención Temprana, etc.).

En la Tabla 1, se recogen los datos fundamentales de las guías utilizadas. Algunas de ellas reconocidas internacionalmente, como el caso de NICE o SIGN (Lauritsen, 2013).

Tabla 1. Selección de Guías de Buenas Prácticas

Autor Nombre Año

Filipeck et al.

Practice parameter: Screening and diagnosis of au- 2000 tism: Report of the Quality Standards Subcommittee of the American Academy of Neurology and the Child Neurology Society

National Autistic Society: Ann National Autism Plan for Children (NAPC) 2003 Le Couteur, Chair, Core Working Group

Grupo de Estudios TEA del Instituto de Salud Carlos III (Diez- TEA Cuervo et al., 2005)

Scottish Intercollegiate Guidelines Network (SIGN)

Assessment, diagnosis and clinical interventions for 2007 children and Young people with autism spectrum disorders.

Ministries of Health and Educa- New Zealand Autism Spectrum Disorder Guideline 2008 tion

Sistema Nacional de Salud Guía de Práctica Clínica para el Manejo de Pacien- 2009 (SNS) tes con TEA en Atención primaria

National Institute for Health and Autism:recognition, referral and diagnosis of chil- 2011 Care Excellence (NICE) dren and Young people on the autism spectrum.

Autism diagnosis in children and young people 
El análisis de estas publicaciones parte de la estructura definida por una concepción comprehensiva y educativa del diagnóstico, con el objetivo de servir de referencia para la práctica profesional. De este modo, se aborda la comparación y síntesis en seis aspectos fundamentales: el proceso diagnóstico, los objetivos, los profesionales, las áreas de exploración, los instrumentos, las familias y la intervención.

\section{El Proceso Diagnóstico}

Al hablar de proceso, nos referimos a la secuencia de fases en la que se enmarcan los restantes componentes del diagnóstico y su praxis. La descripción de esta secuencia puede variar en el número de pasos incluidos, en la mayor o menor concreción de los tiempos de ejecución, y en el grado de desarrollo de los subprocesos implícitos en la estructura general.

El estándar diagnóstico para la práctica establece un proceso multidisciplinar y multiagencia, en el que se incorpora a la familia, y que parte de un diseño de actuación en el que la información es recopilada, contrastada y validada.

Desde la detección y posterior derivación, se determina la pertinencia de la evaluación y su naturaleza, se identifican previamente las áreas a evaluar, y presenta el diagnóstico no cómo una mera recogida de datos sino como un esfuerzo de validación de la información con miras a la intervención. El proceso se plasma en un documento escrito que, a su vez, permite la comunicación y coordinación de los diferentes profesionales.

\section{Los Objetivos}

Todo proceso diagnóstico perseguirá unos objetivos particulares y circunscritos a la persona y su contexto, así como a la finalidad de la propia organización a la que pertenece el profesional.

Pero también existen unos objetivos generales desde los que esa concreción debe partir. Los objetivos generales definidos por la National Autistic Society NAPC (2003), son asumidos a su vez por la New Zealand Autism Spectrum Disorder Guideline (2008), y completados con alguna puntualización procedente del Instituto de Salud Carlos III, recogen las ideas básicas presentes en las diferentes guías:

- Identificar, con la mayor fiabilidad posible, las necesidades de salud del niño, incluyendo el diagnóstico diferencial, etiología y consejo genético.

- Promover la comprensión y acuerdo acerca de las implicaciones potenciales del trastorno para el desarrollo, de forma que puedan implementarse estrategias efectivas de tipo educativo, conductual, emocional, comunicativo, social y físico.

- Situar las necesidades del niño en el contexto de la familia, dándoles confianza para responder a ellas y entendiendo que las suyas propias (incluyendo necesidades culturales y espirituales) son también tenidas en cuenta.

Y, todo ello, siendo conscientes de que los datos aportados pueden contribuir a la investigación científica.

\section{Los Profesionales}

Las recomendaciones desde las buenas prácticas respecto a los profesionales se basan fundamentalmente en dos aspectos, uno referido a los equipos y su composición, y otro res- 
pecto a las necesidades de especialización, en formación y experiencia. La totalidad de las guías consultadas apuesta por un equipo multidisciplinar encargado del diagnóstico. Su composición puede variar según el perfil profesional/académico de sus miembros pero, en términos generales, se incluyen profesionales de la psicología, medicina, educación y especialidades como terapia ocupacional, fisioterapia, enfermería o psicoterapeutas.

Desde la composición de los equipos, destaca la propuesta de una figura esencial por las funciones que le competen: la creación de una figura de coordinación del proceso, un trabajador clave (NAPC 2003), coordinador del caso (NICE 2011; New Zealand, 2008) o facilitador (SNS, 2009). Su presencia permite dotar de coherencia al proceso desde la perspectiva de la familia y de los propios profesionales implicados.Según algunos autores, este papel podría ser asumido por los profesionales de trabajo social (Casey y Elswick, 2011).

\section{Las Áreas de Exploración}

Las categorías de información sobre las que focalizar nuestra atención para la definición del perfil de necesidades y adopción de decisiones de toda índole es, quizás, el apartado que más diferencias plantea entre las diferentes propuestas. Si bien en todas ellas se enfatiza el carácter comprehensivo de la exploración, intentando abarcar la totalidad del individuo y su contexto, no siempre se abordan con la misma amplitud, y difieren en su especificidad y correspondencia con los criterios diagnósticos de TEA.

Respecto a la globalidad, las principales divergencias se refieren a la inclusión o no de áreas como dinámica y entorno familiar, contextos no familiares, motricidad, funcionamiento en vida diaria (más allá de capacidades o habilidades), salud mental y aspectos emocionales. Preocupaciones de los padres aparece también como área de exploración más que como información adicional o como parte de la anamnesis previa.

El otro aspecto diferenciador es el grado en que los TEA y sus alteraciones, o criterios de clasificación, determinan las áreas de exploración. El NICE (2011, 2013) distingue entre las áreas para el diagnóstico de TEA (centradas en los criterios de ICD-10 o DSM-IV) y aquellas necesarias para la obtención de un perfil de la persona con TEA. Esta distinción no está presente en las restantes guías.

Más allá de las diferencias, el estándar de calidad para la práctica respecto a las áreas de exploración se concretaría en tres puntos:

- Evaluación comprehensiva, que incluya diferentes áreas y contextos, más allá de las necesarias para confirmar o refutar criterios clasificatorios cerrados y preestablecidos.

- Las áreas deben ser determinadas antes de comenzar el proceso (NICE, 2011, 2013).

- Como áreas convenientes y necesarias podrían incluirse:

Familia (preocupaciones y señales de alarma)

Funcionamiento diario en diferentes contextos

Síntomas TEA (según criterios de ICD-10 o DSM-IV) en Habilidades Sociales, Comunicativas y Conducta 
Historial médico

Examen Físico

Habilidad intelectual y estilo de aprendizaje

Habilidades académicas

Habla, lenguaje y comunicación

Habilidades de motricidad gruesa y fina; Conducta adaptativa (incluyendo autocuidado)

Salud mental y emocional (incluyendo autoestima)

Nutrición y salud física

Sensibilidad sensorial

Conductas que puedan alterar el funcionamiento diario y participación social

Habilidades de socialización

Comorbilidades.

En todo caso, debemos resaltar que ofrecer un listado de áreas puede ser arriesgado, puesto que parece implicar una visión compartimentada de la persona. Así, debemos añadir a este estándar la visión interactiva que la Organización Mundial de la Salud plantea en su Clasificación Internacional del Funcionamiento, de la Discapacidad y de la Salud (CIF-IA) (OMS, 2011), al hablar de la discapacidad como resultado de la interacción entre las personas que sufren algún tipo de trastorno, enfermedad o causa física, y los factores personales y ambientales. Con frecuencia, el foco se localiza en las limitaciones de funciones y estructuras corporales, y olvida que el entorno puede limitar las actividades y restringir la participación de las personas con TEA, más que el propio trastorno en sí (Durkin, 2013). Las necesidades de las personas con TEA derivarán de esta dinámica, y por tanto, las áreas de exploración para el diagnóstico, deben atender a esta interacción.

Complejidad, comprehensividad y totalidad, son conceptos que se derivan de esta visión y que sólo pueden entenderse desde el juego de todos estos factores y variables, no cómo compartimentos aislados.

\section{Instrumentos}

Los métodos e instrumentos de recogida de información constituyen otro de los apartados que más diferencias presenta entre las diferentes propuestas y en la propia práctica. Las recomendaciones varían según el grado de concreción del listado, la especificidad para el TEA de los instrumentos propuestos, el carácter más o menos cualitativo/cuantitativo de los mismos y los criterios psicométricos de selección.

Como estándar de calidad para la práctica se plantean, más que listados prefijados, cuatro ideas básicas:

- Utilización de instrumentos estandarizados, especialmente aquellos dirigidos a la especificación de la presencia de TEA y áreas cognitiva, comunicación y adaptativa; y no estandarizados, incluyendo, observación directa en diferentes contextos y en situa- 
ciones estructuradas y no estructuradas, entrevista familiar, revisión de documentos, y tareas no estandarizadas.

- Considerar los criterios de bondad de las pruebas estandarizadas. En el caso concreto de aquellas específicas de TEA, con unos intervalos de sensibilidad y especificidad determinados.

- Contraste de fuentes en la recogida de información y aplicación de instrumentos.

- No utilizar un único instrumento, sino una batería de instrumentos junto a juicio clínico.

\section{Familias}

La posición que las guías adjudican a la familia durante el proceso diagnóstico es muy diferente, yendo desde su papel como informadores hasta situarlos como centro del propio proceso. En nuestro análisis se reconocen 4 roles fundamentales:

- Informadores: las referencias se centran en su papel a la hora de recoger información sobre el desarrollo o detectar señales de alarma.

- Usuarios: con esta definición se recogen aquellas recomendaciones que, si bien se centran en el bienestar de las familias en el proceso, no llega a situarlos como un objeto específico del diagnóstico. En este caso se habla de cómo comunicar los resultados, los valores del profesional en el contacto con la familia o aportar información como estrategia de apoyo.

- Objeto de diagnóstico e intervención: en este caso la familia figura como área de exploración y como objeto del plan de actuación elaborado.

- Coterapeutas: de una manera explícita reconoce su papel en la intervención y dispone recomendaciones respecto a estrategias de apoyo en este sentido.

Como estándar de calidad para la práctica destacan los cuatro roles asignados a la familia desde el NAPC (2003) sin excepción, valorando su papel como informadores, coterapeutas, objeto de diagnóstico e intervención. Como protagonistas también del proceso, cada etapa del mismo debe planificarse y discutirse con las familias, informándoles de todos los pasos y resultados. La información y la comunicación (en su contenido y en los valores de respeto, confidencialidad, transparencia y compromiso) es parte fundamental como estrategia de apoyo, pero éste debe también contemplar su necesidad de entrenamiento en pautas educativas y de relación con su hijo/a y su capacidad de decisión en el plan de actuación establecido. Este apoyo debe adaptarse a los diferentes momentos del proceso y aún más en los períodos de transición

\section{Intervención}

Exceptuando dos guías -NICE (2011) y SNS (2009)-, en las que no constan referencias a la intervención, ésta se presenta desde los estándares de calidad, bien como una parte integrante del proceso diagnóstico (SIGN, 2008; NAPC, 2003) bien como un plan basado en los resultados obtenidos, aunque no se explicite su inclusión en el propio proceso.

También el rol de la familia en la intervención es un elemento diferenciador entre las diferentes propuestas. Aquellas que la incluyen, la consideran como un objeto de actuación en 
sí misma (NAPC, 2003), planteando intervenciones específicas que respondan a sus necesidades, o atendiendo a su papel como gestores y coordinadores de recursos y profesionales para sus hijos.

Las cautelas sobre la idoneidad de los estándares de calidad para el diagnóstico en educación, cobran en este apartado su máxima importancia. Aunque algunas de las guías, como el NAPC (2003), incluyen la intervención en el propio proceso diagnóstico, las restantes se limitan a descripciones más o menos generales de las diferentes opciones o, en el mejor de los casos, describen las diversas opciones a modo de listado. Para poder tener una visión general sobre la intervención en TEA desde la perspectiva educativa, es necesario completar las aportaciones desde las buenas prácticas con otros trabajos que aborden la temática teniendo en cuenta la evidencia científica y el abordaje práctico de la misma.

Desde la clasificación de Mesibov, Adams y Klinger (1997), recogida también en la guía práctica publicada por el Departamento de Salud Australiano (Prior, Roberts, Rodger, Williams, y Sutherland, 2011; Roberts y Prior, 2006), las intervenciones pueden diferenciarse entre biológicas, psicodinámicas y educativas. El desarrollo de cada una de estas categorías excede al objetivo de este artículo pero sí es necesario atender a aquellas susceptibles de implementarse en los servicios educativos como consecuencia y parte de los procesos diagnósticos, las intervenciones educativas.

Saldaña y Moreno (2012) resumen las recomendaciones fundamentales para la puesta en práctica de las intervenciones educativas en los siguientes puntos:

- Las intervenciones que incorporan elementos básicos de procedimientos conductuales son las que acumulan más evidencias. Estos deben aplicarse a problemas conductuales, adoptando fundamentalmente el apoyo conductual positivo, así como el control de estímulos ambientales.

- Respecto a programas combinados y específicos/focalizados recomiendan los sistemas de estructuración y apoyos visuales del programa TEACCH junto al Early Start Denver Model, PECS e Historias Sociales en casos con perfil adecuado a sus características, y los sistemas alternativos o aumentativos de comunicación.

- En el tiempo de intervención, se recomienda una intensidad relativamente alta, incluso de 20 a 25 horas semanales, especialmente en atención temprana.

- El entrenamiento y colaboración familiar constituye un elemento positivo para todos los implicados.

- Finalmente, el uso de terapias psicodinámicas, sensoriomotoras o asistidas con animales no aparecere comendada por los autores. Por otro lado, la musicoterapia o integración sensorial necesitan acumular mayor evidencia empírica.

La aplicación de estos principios en la práctica ha sido analizada por Saldaña, Sarmiento, Moreno y Hernández (2010) en el contexto de una investigación sobre las intervenciones educativas en personas con TEA por parte de las administraciones públicas. Sus resultados apuntan que, aún a pesar de que los principios de las buenas prácticas reciben una alta valoración por parte de los profesionales, su implementación en la práctica no se correspondía 
en intensidad y especificidad a los estándares de calidad. La conclusión última de los investigadores nos parece relevante y de especial interés para este artículo de análisis sobre la práctica diagnóstica y los estándares de calidad. Aunque hace referencia directa a la intervención esta reflexión posee una clara conexión con el conjunto del proceso diagnóstico:

"Parece claro que se requiere un esfuerzo para ampliar la base específica de conocimientos que los centros tienen sobre la efectividad de las terapias con TEA disponibles. Programas formativos o la mera difusión de revisiones sistemáticas pueden resultar de utilidad en un primer momento para ello. En el caso de la implementación efectiva de algunos componentes, sin embargo, serán necesario actuaciones más centradas en la aplicación real de las diferentes estrategias propuestas"(Saldaña et al., 2010, p.6).

\section{La aplicación de los estándares de calidad en el contexto educativo}

La conclusión de Saldaña et al. (2010) deja abierta la puerta a una reflexión fundamental para el profesional y su praxis en el contexto educativo: ¿cómo pueden llevarse a cabo las recomendaciones de los estándares de calidad?

Un primer paso podría consistir en abordar estos modelos de buenas prácticas desde una propuesta específica para el ámbito educativo entendido de modo amplio, no únicamente restringido a las escuelas y a la llamada educación formal. Desde la educación, el TEA es sólo un componente más de un conjunto de necesidades que varían según la intervención y las fortalezas del niño. Por tanto, el proceso de diagnóstico se debe caracterizar por ese sentido global y comprehensivo en el que la intervención es parte integrante y fundamental (Arnaiz y Zamora, 2012; Palomo, 2013). Siguiendo a Marí (2007, p. 37) "diagnosticar en Educación no pretende demostrar o falsar hipótesis o conjeturas, sino encontrar soluciones correctivas o proactivas, para prevenir o mejorar determinadas situaciones de los sujetos en orden a su desarrollo personal en situaciones de aprendizaje".

Por tanto, elementos como intervención y familia, con presencia dispar en las anteriores guías de buenas prácticas, aparecen como elementos fundamentales desde un modelo educativo del diagnóstico. La selección de áreas de exploración e instrumentos necesitan, aún más si cabe, de un enfoque comprehensivo e integrador, que recoja esas fortalezas y necesidades dinámicas y cambiantes. Un modelo educativo incluye, además, las situaciones en las que la persona se encuentra para encontrar soluciones. No sólo se trata de buscar una etiqueta sino de abordar la globalidad.

El Diagnóstico en Educación puede recoger las aportaciones de los estándares de calidad y proporcionar un marco adaptado para el contexto educativo. Más concretamente, a través una propuesta que se plantea como un modelo de diagnóstico integrador y compatible con la investigación científicamente fundada. Marí (2006, 2007; Marí, Climent, y Cervera, 2009) definió este modelo como Diagnóstico Pedagógico "considerado como un proceso de indagación científica, apoyado en una base epistemológica y cuyo objeto lo constituye la totalidad de los sujetos (individuos o grupos) o entidades (instituciones, organizaciones, programas, contextos familiar, socio-ambiental, etc.) consideradas desde su complejidad y abarcando la globalidad de su situación, orientado a la comprensión y valoración de cual- 
quier hecho educativo y que incluye en su proceso metodológico, necesariamente, una intervención educativa de tipo preventivo-perfectivo" (Marí, 2006, p. 201).

\section{Las fases del proceso diagnóstico}

Desde esta perspectiva, el proceso de diagnóstico incorpora 5 fases fundamentales, compuestas, a su vez, de diferentes pasos interconectados, alejándose de una concepción meramente lineal (ver Figura 1).

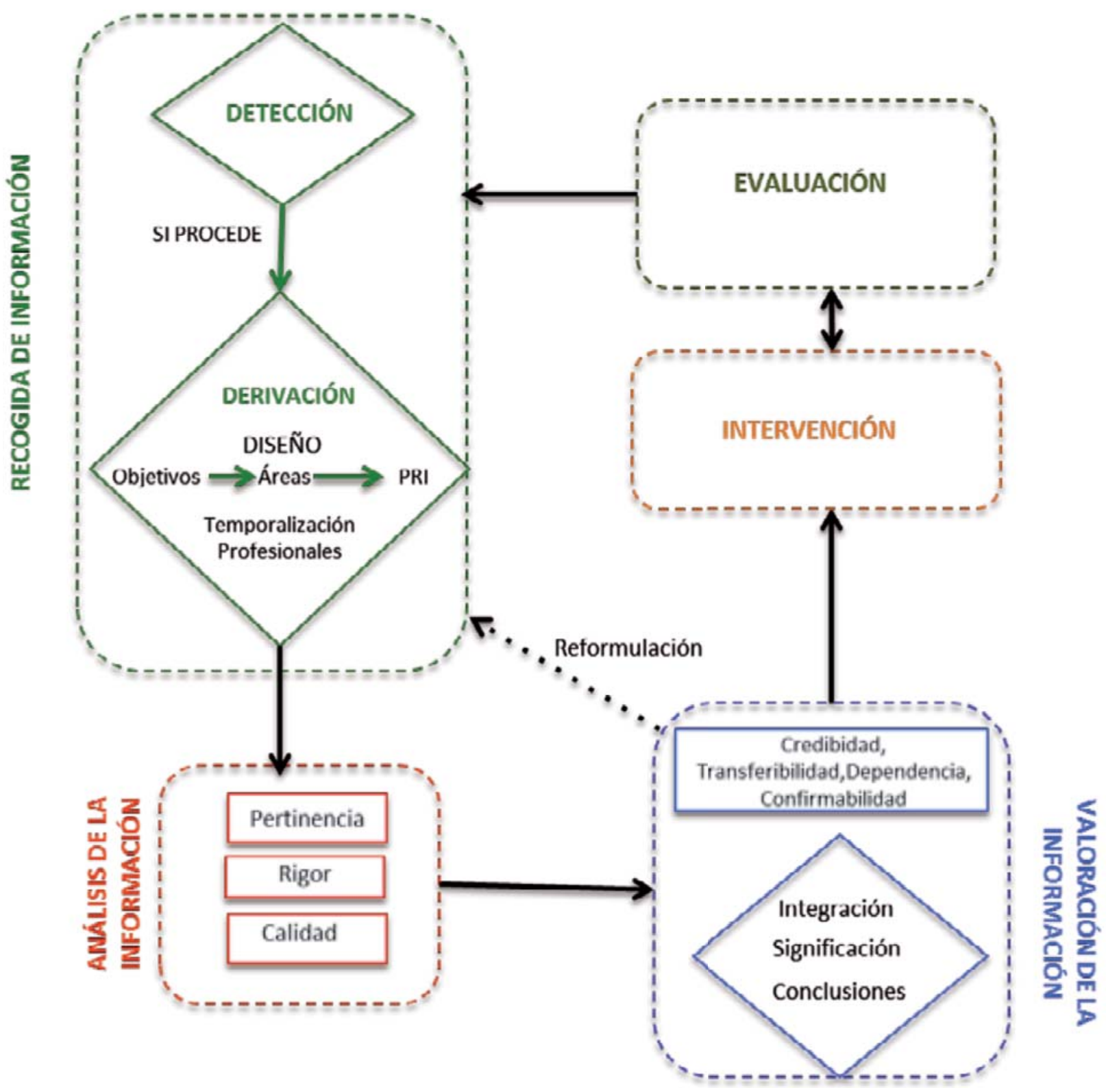

Figura 1. Secuencia del Proceso Diagnóstico

La primera fase se compone de tres acciones complementarias: la detección, la derivación y la recogida de información. Con la detección se recoge toda la información disponible para identificar el problema y resolver la continuidad o no del proceso. En caso 
afirmativo entraría la derivación, momento en el cual se establecen los objetivos, áreas de exploración,procedimientos de recogida de información (PRI), temporalización y profesionales implicados, en resumen el diseño global del proceso. Éste será además informado a las familias. La puesta en marcha del diseño comienza con la recogida de información. Este paso parte de tres premisas fundamentales: la multidimensionalidad del objeto diagnóstico, la complementariedad metodológica y paradigmática, asumible desde lo que podría considerarse metodológicamente un enfoque mixto, y la multidisciplinariedad de los profesionales responsables.

En la siguiente fase, análisis de información, el profesional explicita y analiza la adecuación de los instrumentos/pruebas, estrategias seguidas para conseguir la precisión de la medida en términos de pertinencia de su selección para el diagnóstico e intervención, el rigor de su administración y la calidad de los instrumentos empleados. Se cierra con la exposición de los resultados obtenidos.

Siguiendo con la secuencia del proceso diagnóstico, la valoración de información implica la síntesis de los datos expuestos, de forma integrada y complementaria, atendiendo a la significación de los resultados, detectando contradicciones y buscando la resolución de las mismas. Para finalmente establecer conclusiones y reformular los objetivos iniciales de exploración si fuera preciso.

Basada en estas conclusiones se establece una propuesta de intervención, desde la que se valida el propio proceso diagnóstico. Y, finalmente, se propone una evaluación del conjunto del proceso, incluyendo a todos los agentes implicados y que permite, en cualquier momento, revisar las decisiones adoptadas.

El modelo aporta una visión multidimensional y comprehensiva del diagnóstico que obliga al análisis de todas las variables implicadas y sus interrelaciones, no con un fin descriptivo o clasificatorio, sino para encontrar las razones que permitan una intervención para la mejora de la situación existente (Marí, 2007).Como responsable del proceso diagnóstico, el profesional se ubica como un investigador en la práctica. Desde la complementariedad metodológica y teórica, diseña y ejecuta un plan ad hoc para esa persona en ese entorno y en ese momento, y utiliza los recursos de la ciencia para dotar de rigurosidad y validez sus elecciones y conclusiones.

Pero, retomando la pregunta inicial de nuestro apartado -¿cómo pueden llevarse a cabo las recomendaciones de los estándares de calidad?-, la adopción de un modelo diagnóstico propuesto desde la educación puede suponer un primer paso, pero quizás no sea suficiente. De otro modo, entre el conocimiento basado en la evidencia y su implementación práctica parece existir un agujero todavía difícil de salvar (Allen, Robins y Decker, 2008; Odom, Cox y Brock, 2013; Reichow, Volkmar, y Cicchetti, 2007; Wood, McLeod, Klebanoff, y Brookman-Frazee, 2015). 


\section{Las limitaciones en la práctica profesional}

La conclusión de Saldaña et al. (2010) sobre la implementación de intervenciones educativas específicas de TEA en el contexto escolar señalaba la necesidad de ir más allá de revisiones y difusión de información para conseguir la implementación de los estándares de calidad. Por otro lado, desde las guías revisadas, tanto el juicio experto como la formación y la experiencia del profesional parecen ser las claves para la práctica. Sin embargo, si bien es cierto que estas dos últimas variables aparecen como responsables de las discrepancias en la praxis (Kennedy et al., 2004; Klose et al., 2012; Lord et al., 2011; Sansosti y Sansosti, 2013), otros factores ajenos al profesional también implican importantes diferencias entre servicios y respecto a los estándares de calidad.

De este modo, la percepción de barreras en la organización y en el servicio (Wood et al., 2015) tales como las listas de espera, los límites de tiempo (Kennedy et al., 2004) o las políticas externas e internas de los servicios, puede dificultar la aplicación de las recomendaciones de buenas prácticas. Y también otras racionalizaciones subjetivas de los profesionales pueden obstaculizar su implementación (Kennedy et al., 2004), tales como la sensación de urgencia a la hora de obtener un diagnóstico o el grado de certeza respecto al mismo.

En resumen, no basta con proponer un modelo adaptado para educación. Aspectos organizacionales e individuales también deben ser considerados para entender y optimizar la implementación de modelos de buenas prácticas. Así, se abre paso la necesidad de un esfuerzo de investigación global que parta de la realidad, y no sólo de la evidencia desde el laboratorio, que atienda a las limitaciones de recursos de las organizaciones y sistemas implicados, e incluso las de competencias personales y profesionales de los participantes.

\section{A modo de conclusión y líneas de investigación futura}

El estudio de la práctica diagnóstica debe ser un punto de partida necesario para la puesta en marcha de acciones de mejora y planes generales de calidad. Para ello son necesarias más investigaciones que, en primer lugar, incluyan a todos los servicios y agentes implicados, especialmente a las familias. Y que completen la perspectiva de los participantes, con información recogida a través de entrevistas y grupos de discusión. En el contexto nacional existen antecedentes de investigaciones sobre la integración de la perspectiva profesional y de las familias en la Comunidad de Madrid (Belinchón, 2001) y, más recientemente, desde la Asociación AETAPI -Asociación Española de Profesionales del Autismo- (Pozo et al., 2014).

Por otro lado, también resulta preciso profundizar en los obstáculos que separan a los profesionales de los estándares de buenas prácticas, así como ahondar en las causas e implicaciones de la separación entre diagnóstico e intervención de cara a poder respaldar actuaciones dirigidas a su integración global. Y, finalmente, son necesarias investigaciones que resulten en la elaboración de un estándar de calidad para la educación. Resulta necesario abordar un "mapeo" de la realidad profesional, ahondando en sus necesidades y con una propuesta de intervención específica. Un ejemplo de esta línea de trabajo sería la desarrollada desde el gobierno escocés en su Scottish Strategy for Autism (2011). 


\section{Referencias}

Adelman, C. R. (2010). Factors that influence age of identification of children with autism and pervasive developmental disorders NOS. University of Houston.Disponible en ProQuest Dissertations \& Theses A\&I. (756862835). Recuperado de http://search.proquest.com/ docview/ 756862835 ? accountid $=14777$

Allen, R.A., Robins, D.L., yDecker, S.L. (2008) Autism Spectrum Disorders: Neurobiology and Current Assessment Practices. Psychology in the Schools, 45(10), 905-917. DOI: 10.1002/pits

Arnáiz, J., y Zamora, M. (2012). Detección y Evaluación Diagnóstica en TEA. En Martínez, M.A. y Cuesta, J.L. Todo sobre el Autismo: Los Trastornos del Espectro Autista (TEA) Guía completa basada en la ciencia y en la experiencia(pp. 89-132). Tarragona, España: Publicaciones Altaria.

Balasubramanian, B., Bhatt, C.V.,y Goyel, N.A. (2009). Genetic studies in children with intellectual disability and autistic spectrum of disorders. Indian Journal of Human Genetics, 15(3), 103-107. D0I: 10.4103/0971-6866.60185

Belinchón, M. (coord) (2001). Situación y necesidades de las personas con trastornos del espectro autista en la Comunidad de Madrid. Madrid: Ediciones Martín y Macias.

Blatt, G.J.(Ed.) (2010).The Neurochemical Basis of Autism. FromMolecules to Minicolumns [versión electrónica de SpringerScience + Business Med, LLC]. Boston, USA: Springer. DOI 10.1007/978-1-4419-1272-5

Canal-Bedia, R., García-Primo, P., Hernández-Fabián, A., Magán-Maganto, M., Sánchez, A.B., y Posada-De la Paz, M. (2015). De la detección precoz a la atención temprana: estrategias de intervención a partir del cribado prospectivo. Revista de Neurología, 60 (Supl 1), S25-9.

Casey, J.P., Magalhaes, T., Conroy, J.M., Regan, R., Shah, N., Anney, R. ... Ennis, S., (2012). A novel approach of homozygous haplotype sharing identifies candidate genes in autism spectrum disorder.Human Genetics, 131 (4), 565-579. DOI 10.1007/s00439-011-1094-6

Casey, L.B., yElswick, S.E. (2011) The Social Worker's Role in the Assessment of Autism Spectrum Disorder. Children \& Schools,33 (3), 176 183.

Centers for Disease Control and Prevention (2014). Prevalence of Autism Spectrum Disorder Among Children Aged 8 Years - Autism and Developmental Disabilities Monitoring Network, 11 Sites, United States, 2010. MMWR, 63, 1-21.

Díez-Cuerv,o A., Muñoz-Yunta, J.A., Fuentes-Biggi, J., Canal-Bedia, R., Idiazábal-Aletxa, M.A., Ferrari-Arroyo, M.J., ... Posada-De la Paz , M. (2005). Guía de buena práctica para el diagnóstico de los trastornos del espectro autista. Revista de Neurología, 41 (5), 299-310.

Durkin, M. (2013). The Epidemiology of Autism Spectrum Disorder: Toward a More Inclusive World [Video]. Disponible en: https://imfar.confex. com/imfar/2013/videogateway.cgi/id/335? recordingid = 335

Fernell, E., Eriksson, M.A., y Gillberg, C. (2013). Early diagnosis of autism and impact on prognosis: a narrative review. Clinical Epidemiology,5, 33-43. DOl.org/10.2147/CLEP.S41714

Filipek, P.J., Accardo, S., Ashwal, Baranek, G.T., Cook, E.H., Dawson, G., ... Volkmar, F.R. (2000). Practice parameter: Screening and diagnosis of autism: Report of the Quality Standards Subcommittee of the American Academy of Neurology and the Child Neurology Society. Neurology, 55, 468. DOI 10.1212/WNL.55.4.468

Fountain, C., King, M.D.,yBearman, P.S.(2011) Age of diagnosis for autism: individual and community factors across 10 birth cohorts. Journal of Epidemiology and Community Health, 65, 503-510. D0I:10.1136/jech.2009.104588

Gillberg, C. (2010). The ESSENCE in child psychiatry: Early Symptomatic Syndromes Eliciting Neurodevelopmental Clinical Examinations. Research in Developmental Disabilities, 31, 1543-1551. D0I:10.1016/j.ridd.2010.06.002

Güemes, I., Martín, M.C., Canal, R. y Posada-de la Paz, M.(2009). Evaluación de la eficacia de las intervenciones psicoeducativas en los trastornos del espectro autista. Madrid: Instituto de Investigación de Enfermedades Raras - Instituto de Salud Carlos III, Noviembre de 2009. Recuperado de http://www.ite.educacion.es/formacion/materiales/185/cd/unidad_4/material_m4/Evaluacion_de_la_ eficacia IPTEA.pdf

GuiaSalud [Internet]. Guía de Práctica Clínica para el Manejo de Pacientes con Trastornos del Espectro Autista en Atención Primaria. [Actualizada el 07 de mayo de 2010. Citada el 4 de junio de 2010]. Recuperado de: http://www.guiasalud.es/egpc/autismo/completa/ index.html 
Kennedy, T., Regehr, G., Rosenfield, J., Roberts, W., y Lingard,L. (2004). Exploring the Gap Between Knowledge and Behavior: A Qualitative Study of Clinician Action Following an Educational Intervention. Academic Medicine, 79(5), 386-393.

Klin, A., Klaiman, C., y Jones, W. (2015). Rebajar la edad de diagnóstico del autismo: la neurociencia del desarrollo social afronta un importante problema de salud pública. Revista de Neurología, 60 (1), 3-11.

Klose, L. M., Plotts, C., Kozeneski, N., y Skinner-Foster, J. A. (2012). Review of Assessment Tools for Diagnosis of Autism Spectrum Disorders: Implications for School Practice. Assessment for Effective Intervention,37(4), 236-242. D0I: 10.1177/1534508411415090

Leblond, C.S., Heinrich, J., Delorme, R., Proepper, C., Betancur, C., Huguet, G., ...Bourgeron, T. (2012) Genetic and Functional Analyses of SHANK2 Mutations Suggest a Multiple Hit Model of Autism Spectrum Disorders. PLoS Genetics, 8(2), e1002521. D0I:10.1371/ journal.pgen. 1002521

Le Couteur, A., Baird, G., Mills, R., Jordan, R., Howlin, P., Hollins, S. ... O'Sullivan, T. (2003). National Autism Plan for Children (NAPC): Plan for the Identification, Assessment, Diagnosis and Access to Early Interventions for Pre-School to Primary School Aged Children with Autism Spectrum Disorders (ASD). London (United Kingdom): The National Autistic Society. Recuperado de: http://www.autism. org.uk/About-autism/Our-publications/Reports/Otherreports/The-National-Autism-Plan-for-Children.aspx

Lord, C., Petkova, E., Hus, V., Gan, W., Lu, F., Martin, D.M., Ousley, O., Guy, L.,... Risi, S. (2011).A Multisite Study of the Clinical Diagnosis of Different Autism Spectrum Disorders.Archives Of General Psychiatry,69 (3), 306-313. D0I: 10.1001/archgenpsychiatry.2011.148

Marí, R. (2006) Diagnóstico Pedagógico: Un modelo para la intervención psicopedagógica. 2ªd. Barcelona, España: Ariel Educación.

Marí, R. (2007). Propuesta de un modelo de diagnóstico en educación. Bordón,59 (4), 611-626.

Marí, R., Climent, C., y Cervera, M.A. (2009) Guía de Buenas Prácticas para el Diagnóstico Pedagógico. Valencia, España: Tirant Lo Blanch.

Mesibov, G. B., Adams, L. W., yKlinger, L. G. (1997). Autism: Understanding the disorder. New York: Plenum Press.

Ministries of Health and Education (2008).New Zealand Autism Spectrum Disorder Guideline.Ministry of Health, Wellington.Recuperado de: http://www.health.govt.nz/publication/new-zealand-autism-spectrum-disorder-guideline

Mulas, F., Ros-Cervera, G., Millá, M.G., Etchepareborda, M.C., Abad, L. y Téllez de Meneses, M. (2010). Modelos de intervención en niños con autismo. Revista de Neurología, 50 (3), 77-84. Disponible en: http://www.neurologia.com/pdf/Web/50S03/bdS03S077.pdf

National Collaborating Centre for Women's and Children's Health (UK) (2011). Autism: Recognition, Referral and Diagnosis of Children and Young People on the Autism Spectrum. London: RCOG Press, 2011 Sep. (NICE Clinical Guidelines, No. 128.). Disponible en : http:// www.ncbi.nlm.nih.gov/books/NBK92985/

National Institute for Health and Care Excellence (NICE).The management and support of children and young people on the autism spectrum. NICE clinical guideline 170. London, UK: NICE; (2013).

Noterdaeme, M.,y Hutzelmeyer-Nickels, A. (2010).Early symptoms and recognition of pervasive developmental disorders in Germany. Autism, 14, 575. DOI: $10.1177 / 1362361310371951$

Nygren, G., Cederlund, M., Sandberg, E., Gillstedt, F., Arvidsson, T., Gillberg, I.C., Andersson, G.W., yGillberg, C. (2011). The Prevalence of Autism Spectrum Disorders in Toddlers: A Population Study of 2-Year-Old Swedish Children. Journal of Autism and Developmental Disorders, 42(7), 1491-7. DOI 10.1007/s 10803-011-1391-x

Odom, S.L, Cox, A. W. y Brock, M. E. (2013). Implementation Science, Professional Development, and Autism Spectrum Disorders.ExceptionalChildren, 79(2), 233-251.

Organización Mundial de la Salud (OMS) (2011). Clasificación Internacional del Funcionamiento, de la Discapacidad y de la Salud: versión para la infancia y adolescencia: CIF-IA. Madrid: Ministerio de Sanidad, Política Social e Igualdad Centro de Publicaciones

Pozo, P., Zamora, M., Alonso, R., Illera, A., Marquez, C., Maseda, P., Perez, I., Rodriguez, J. y Tendero, R.(2014). Estudio sobre las relaciones de colaboración entre profesionales y familias de personas con trastorno del espectro del autismo (TEA): la perspectiva de los profesionales. Siglo Cero: Revista Española sobre Discapacidad Intelectual. 45 (2) 250, 19-56.

Prior, M., Roberts J.M.A., Rodger, S., Williams, K., y Sutherland, R. (2011). A review of the research to identify the most effective mode/s of practice in early intervention of children with autism spectrum disorders.Department of Families Community Services and Indigenous Affairs, Australia.Recuperado de: https://www.dss.gov.au/our-responsibilities/disability-and-carers/program-services/ for-people-with-disability/full-review-report

Reichow, B., Volkmar, F.R., y Cicchetti , D.V. (2008). Development of the Evaluative Method for Evaluating and Determining Evidence-Based Practices in Autism. Journal of Autism and Developmental Disorders, 38, 1311-1319 .D0I 10.1007/s 10803-007-0517-7 
Roberts, J. M. A.,y Prior, M. (2006).A review of the research to identify the most effective models of practice in early intervention of children with autism spectrum disorders. Australia: Australian Government Department of Health and Ageing. Recuperado de: http://www. health.gov.au/internet/ main/publishing.nsf/ Content/mental-pubs-r-autrev

Saldaña, D., Sarmiento, V. , Moreno, F.J., y Hernández, A. (2010). Un estudio sobre la aplicación de "buenas prácticas" en la atención temprana a niños y niñas con TEA en Andalucía. Comunicación presentada en el XVCongreso Nacional de Autismo AETAPI, Zaragoza. Recuperado de http://aetapi.org/?wpdmdl= 883

Saldaña, D., y Moreno, F.C. (2012). Eficacia de las intervenciones de naturaleza psicológica en los TEA: Evidencias disponibles. En Martínez, M.A. y Cuesta, J.L.(Eds.).Todo sobre el Autismo: Los Trastornos del Espectro Autista (TEA) Guía completa basada en la ciencia y en la experiencia, pp. 191-22). Tarragona: Altaria.

Salvadó-Salvadó, B., Palau-Baduell, M., Clofent-Torrentó, M., Montero-Camacho, M., Hernández-Latorre, M.A. (2012). Modelos de intervención global en personas con trastorno del espectro autista. Revista de Neurología,54 (1), 63-71.

Sansosti, F.J., y Sansosti, J.M. (2013). Effective school-Based service delivery for students with Autism Spectrum Disorders: where we are and where we need to go. Psychology in the Schools, 50(3), 229-244. D0I: 10.1002/pits.21669

Simonoff, E. (2012). Autism spectrum disorder: prevalence and cause may be bound Together. The British Journal of Psychiatry,201, 88-89. DOI: 10.1192/bjp.bp.111.104703

Scottish Intercollegiate Guidelines Network (SIGN) (2007). Assessment, diagnosis and clinical interventions for children and young people with autism spectrum disorders.Edinburgh, Scotland. Recuperado de: http://www.sign.ac.uk/guidelines/fulltext/98/

Volkmar, F.R., y Pauls, D.(2003). Autism. The Lancet, 362 (9390), 1133-1141

Wood, J.L., MCLeod, B.D., Klebanoff, S.,y Brookman-Frazee,L. (2015). Toward the implementation of evidence-based interventions for youth with Autism Spectrum Disorders in schools and community agencies. Behavior Therapy, 46, 83-95.D0l:10.1016/j. beth.2014.07.003

Zimmerman, A.W. (Ed.) (2008). Autism: Current Theories and Evidence.Baltimore: Springer. D0I: 10.1007/978-1-60327-489-0 